\title{
ON SEMIPRIME JORDAN RINGS $H(R)$ WITH ACC
}

\author{
DANIEL J. BRITTEN 1
}

\begin{abstract}
Let $R$ be a 2-torsion free semiprime associative ring with involution*. Conditions are put on the Jordan ring $H(R)$ of symmetric elements which imply the existence of a ring of quotients which is a direct sum of involution simple Artinian rings. Using this result we obtain a Jordan ring of quotients for $H(R)$.
\end{abstract}

In [1], the author considered 2-torsion free associative rings $R$ with involution * such that the Jordan subring $H$ of symmetric elements $\left(H \subseteq R^{+}\right)$ was prime and satisfies a chain condition on quadratic ideals. In this case it was found that if $R$ was semiprime then $R$ had a ring of quotients which was involution simple Artinian. This result yields a Jordan ring of quotients for $H$ which fits into the second structure theorem [4, p. 179], and for this implication the semiprimeness of $R$ need not be assumed.

Our intention here is to consider the case when $H$ is semiprime and satisfies ACC on quadratic ideals. It is clear that our endeavor is similar to that of extending Goldie's theorem from the prime to the semiprime case and our proof is motivated by this.

Let $M=\Sigma\{A: A$ is a *ideal of $R$ and $A \cap H=0\}$ so that $M^{3}=0$ since it is contained in the skew elements. Thus a semiprime assumption on $R$ gives $M=0$. We shall use $T$ to denote $R / M$. One can easily see that $T$ is 2-torsion free with involution $*$ given by $(x+M) *=x^{*}+M$.

Throughout we shall assume $H(R)$ is a semiprime Jordan ring satisfying the ascending chain condition (i.e. ACC) on quadratic ideals. For the definitions used in this paper please see [1].

Proposition 1. $T$ is semiprime and $H(T)=\left\{\bar{x} \in T: \bar{x}^{*}=\bar{x}\right\}$ is a semiprime Jordan ring with ACC on quadratic ideals.

Proof. We shall prove only that $T$ is semiprime. Suppose $A$ is an ideal

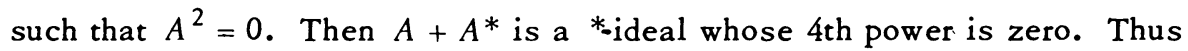

Received by the editors May 7, 1973.

AMS (MOS) subject classifications (1970). Primary 17C10; Secondary 16A28.

1 This research has been supported by National Research Council Grant A-8471, while the author was at the University of Windsor. 
$\left(A+A^{*}\right) \cap H(T)=0$ by semiprimeness of $H(T)$. Hence $A+A^{*}=0$ since every nonzero *ideal of $T$ contains nonzero symmetric elements.

Since our main interest is in obtaining a Jordan ring of quotients for $H$ and $H$ injects into $H(T)$, we shall work inside the semiprime ring $T$. It is clear that a Jordan ring of quotients for $H(T)$ is a Jordan ring of quotients for $H$.

If $S$ is a subset of $T$, then the left annihilator of $S,\{x \in T: x S=0\}$ shall be denoted by $A_{l}(S)$. Similarly we use $A_{r}(S)$ for the right annihilator of $S$.

Lemma 2. (i) If $B$ is a left ideal of $T$ and $A=A_{l}(B)$ then $B A=0$.

(ii) Left annibilators of *ideals are *-ideals.

Proof. (i) $B A T B A=0$ so that $T B A$ is an ideal whose square is zero.

(ii) is clear.

Let $B \neq T$ be a left annihilator of $T$ which is a *ideal such that $B \cap$ $H(T)$ is maximal as compared to similarly defined objects. Let $\beta$ be the set of all such $B^{\prime}$ s. One should notice that $\beta$ is not empty since $H$ satisfies ACC on quadratic ideals.

Lemma 3. If $B \in \beta$ then $B$ is a *-prime ideal (i.e. T/B a *-prime ring).

Proof. Suppose $C$ and $D$ are *ideals of $T$ and $C D \subseteq B$ so that $C D A=$ 0 where $A^{*}=A$ and $B=A_{l}(A)$. If $D A=0$ then $D \subseteq B$. Thus we suppose $D A \neq 0$.

Now $C(D A+A D)=0$, by Lemma 2. Thus $D A+A D$ is a *-ideal and $C \subseteq A_{l}(D A+A D) \neq T$. But $B \subseteq A_{l}(D A+A D)$ and by maximality of $B \cap H(T)$ we see that $C \cap H(T) \subseteq B \cap H(T)$. Thus any symmetric element in $A C+C A$ is in $B$ and in $A$. However, $B \cap A$ is an ideal which squares to zero and hence $B \cap A=0$. Thus $A C+C A$ is a *-ideal which contains no nonzero symmetric elements. Thus $A C+C A=0$ and $C \subseteq A_{l}(A)=B$.

Lemma 4. Let $B_{1}$ and $B_{2}$ be elements of $\beta$, Then

(i) $B_{1} \neq B_{2}$ implies $B_{1} \not \subset B_{2}$, and

(ii) $B_{1} \neq B_{2}$ implies $A_{l}\left(B_{1}\right) \subseteq B_{2}$.

Proof. Suppose $B_{1} \neq B_{2}$ and $B_{1} \subseteq B_{2}$. Then since $A_{l}\left(B_{2}\right) B_{2} \subseteq B_{1}$, we see that the *-primeness of $B_{1}$ gives $A\left(B_{2}\right) \subseteq B_{1} \subseteq B_{2}$ which implies $A_{l}\left(B_{2}\right)^{2}$ $=0$ and hence $A_{l}\left(B_{2}\right)=0$. However, this implies $B_{2}=T$, since if $B_{2}=A_{l}(A)$ then $A B_{2}=0$ so that $A=0$. However, $B_{2} \neq T$ since $B_{2} \in \beta$, contradiction.

(ii) follows immediately from (i) and Lemma 3.

Lemma 5. $\beta$ is a finite set. 
Proof. Let $B_{1}, \cdots, B_{n}, \cdots$ be the distinct elements in $\beta$. By Lemma

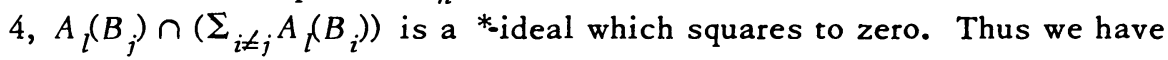
a direct sum $\Sigma A_{l}\left(B_{i}\right)$ and by intersecting the partial sums with $H(T)$ one obtains a strictly increasing sequence of quadratic ideals. Hence, there are only finitely many elements in $B$.

\section{Lemma 6. The elements of $\beta$ intersect at zero.}

Proof. Let $B_{1}, \cdots, B_{n}$ be the elements of $\beta$ and let $I=\bigcap_{i=1}^{n} B_{i}$ so that $\left(\sum_{i=1}^{n} A_{l}\left(B_{i}\right)\right) I=0$. If $A_{l}(I)=T$, then $I^{2}=0$ and hence $I=0$.

Now, we assume $A_{l}(I) \neq T$. This implies that $A_{l}(I) \cap H(T)$ is contained in some $B_{k} \in \beta$ which shows $Q=\left(A_{l}\left(B_{k}\right)\right) \cap(H(T)) \subseteq B_{k} \cap H(T)$. Thus $Q$ is a quadratic ideal such that $U_{Q}(Q)=0$ and hence $Q=0$. But this implies $A_{l}\left(B_{k}\right)$ is zero, which is impossible.

Lemma 6 allows us to consider $T$ as a subdirect sum of the *-prime rings $T_{i}=T / B_{i}, i=1, \cdots, n$. Using the facts that $T$ is 2 -torsion free and $B_{i}$ is an annihilator ideal it is easy to see that $\left\{x \in T: 2 x \in B_{i}\right\}$ is equal to $B_{i}$. Thus $T_{i}$ is 2-torsion free. Also, Erickson and Montgomery [2, Theorem 9] have shown that since $T_{i}$ is a *-prime with involution ${ }^{*}$, the Jordan ring $H\left(T_{i}\right)=\left\{x \in T_{i}: x^{*}=x\right\}$ is prime. $H\left(T_{i}\right)$ satisfies ACC on quadratic ideals for $i=1, \cdots, n$.

By the main theorem of [1], $T_{i}$ has a ring of quotients which is involution simple Artinian. By the discussion on pp. 269 and 270 of [3], we see that $T$ has a ring of quotients which is isomorphic to the direct sum of the ring of quotients of the $T_{i}$ 's.

We have now shown $T$ has a ring of quotients $W$ which is the direct sum of involution simple Artinian rings. One can show, as was done in [1, Theorem 6], that $H(W)$ is a Jordan ring of quotients for $H(R)$.

We now summarize with the following Theorem:

Theorem. Let $R$ be a 2-torsion free associative ring with involution* such that the Jordan ring of symmetric elements $H(R)$ is semiprime and satis. fies ACC on quadratic ideals, and let $M$ be the maximal *ideal of $R$ such that $M \cap H(R)=0$. Then $T=R / M$ has a ring of quotients $W$ which is a direct sum of involution simple Artinian rings. Also, $H(W)$ is a Jordan ring of quotients for $H(R)$.

\section{REFERENCES}

1. D. J. Britten, On prime Jordan rings $H(R)$ with chain condition, J. Algebra 27 (1973), 414-421.

2. T. S. Erickson and M. S. Montgomery, The prime radical in special Jordan rings, Trans. Amer. Math. Soc. 156 (1971), 155-164. MR 43 \#306. 
3. N. Jacobson, Structure of rings, 2nd ed., Amer. Math. Soc. Colloq. Publ., vol. 37, Amer. Math. Soc., Providence, R. I., 1964. MR 36 \#5158.

4. - Structure and representation of Jordan algebras, Amer. Math. Soc. Colloq. Publ., vol. 39, Amer. Math. Soc., Providence, R. I., 1968. MR 40 \#330.

5. C. Lanski, Rings with involution whose symmetric elements are regular, Proc. Amer. Math. Soc. 33 (1972), 264-270. MR 45 \#1971.

6. W. S. Martindale III, Rings with involution and polynomial identities, J. Algebra 11 (1969), 186-194. MR 38 \#3302.

7. Chester Tsai, The prime radical in a Jordan ring, Proc. Amer. Math. Soc. 19 (1968), 1171-1175. MR 37 \#6336.

DEPARTMENT OF MATHEMATICS, UNIVERSITY OF WINDSOR, WINDSOR, ONTARIO, CANADA 